\title{
THE TONOMETRIC SENSOR, A NEW DEVICE FOR THE MEASUREMENT OF INTRAOCULAR PRESSURE
}

\author{
J. A. VOORTHUYZEN, C. DEN BESTEN and P. BERGVELD \\ Twente University, P.O. Box 217, 7500 AE Enschede (The Netherlands)
}

\section{Abstract}

In this paper we present a new sensor for the measurement of intraocular pressure. We have applied the indentation principle, in which the eye is indented by exerting a force on it, while the size of the indented area is monitored. To measure the force we have used a commercial force sensor. The sensor to measure the indentation of the eye has been developed in our laboratories. We have performed measurements using an experimental set-up; these show that an accuracy of $90 \%$ will be possible if the sensor is designed in the proper way.

\section{Introduction}

Tonometry is the measurement of intraocular pressure (IOP). An elevated intraocular pressure, due to a disturbance of the aqueous dynamics such as a decreased outflow, pinches-off blood vessels and reduces the flowthrough of blood in the eye. This may in turn cause the death of optic nerves and result in reduction of vision, or even complete blindness. This indisposition is usually called glaucoma.

Detection of elevated IOP at an early stage and consecutive treatment by ophthalmologists may avoid the occurrence of glaucoma. If, however, glaucoma definitely occurs, recovery is almost impossible. The chance of an elevated IOP is rather large $(2 \%-4 \%)$ for people over 40 , so a regular measurement of their IOP is advantageous.

Present-day set-ups for the measurement of IOP are rather complex and expensive, being limiting to use by specialists only. Most of these systems use mechanical and optical parts to measure IOP. The development of a cheap and easy-to-handle system seems to be attractive, so that even family doctors could regularly perform this measurement. The purpose of the research described here was to develop such a tonometric sensor.

In Section 2 the physical background of the measurement of IOP will be described, by considering the often-used applanation principle. In Section 3 the sensor principle as well as its theoretical behaviour will be described, while in Sections 4 and 5 the experimental results will be presented and discussed. 


\section{The measurement of IOP}

The most accurate and straightforward method of measuring IOP is to use a small catheter with a pressure sensor, placed in the interior of the human eye. For regular measurements however, a non-invasive method is more attractive. A well-known method is the so-called applanation principle. We have decided to apply this principle as well, and therefore it will be discussed in more detail.

The eye is considered to be a liquid-filled spherical container with negligible stiffness. On exerting a force on the eye by means of a probe, it will be indented as shown in Fig. 1. Measuring the applied force $F$ and the indented area $A$ of the eye, the intraocular pressure $P_{10 p}$ can be written as $P_{\text {iop }}=F / A$.

This law was first formulated by Imbert and Fick, and afterwards extended and modified by several others. In particular Goldmann and Schmidt have studied intensively the exact relationship between $P_{\text {iop}}$, $F$ and $A[1,2]$. They have found that two other components have a considerable influence: the rigidity of the cornea and the capillary force of the tear film. They have found that the two terms have opposite influences and cancel each other out approximately if the radius of the indented area is equal to $1.5 \mathrm{~mm}$.

The applanation principle utilizes this fact by adapting the applied force $F$ in such a way that the radius of the indented area is equal to 1.5 $\mathrm{mm}$. This requires the eye to be fixed with respect to the measurement set-up and an adjustable applied force, which is often performed by hand. This is an inconvenient and laborious method of measurement and therefore we have decided to develop a tonometric sensor that continuously measures the applied force as well as the indented area. In this case, a fixation of the eye and the set-up with respect to each other is not required.

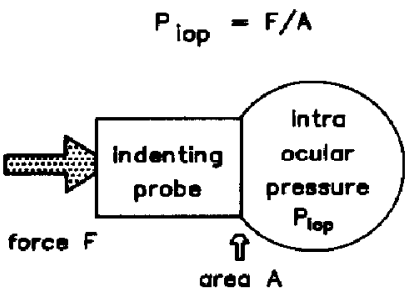

Fig. 1. Cross-section of an eye with internal pressure $P_{\text {iop, }}$ partly externally loaded and indented on an area $A$ with force $F$.

\section{The tonometric sensor}

The combined measurement of the indented area and applied force can be performed in different ways. We have used a commercial force sensor and developed in our laboratory a sensor to measure the size of the indented 
area of the eye. Some of the possible ways of measuring this area directly, via the measurement of resistance or capacitance between the indented eye and the touching electrode that deforms it, have been considered, but finally we have decided to detect the indentation indirectly by using the principle schematically shown in Fig. 2.

The eye is pressed against a thin membrane, metallized on its bottom side. Due to the force applied to the membrane, it is pressed to the rigid support and the eye itself will be indented. The area of the membrane that contacts the support is approximately equal to the indented area of the eye.

The area of the membrane pressed to the support is measured resistively. On the support a lot of parallel metal beams connected as a meander have been realized, as shown in Fig. 3. If the metallized membrane contacts the support, part of the meander will be short-circuited. The decrease of resistance measured between both ends of the meander appears to be linearly proportional to the number of beams short-circuited. Taking the square of this number, we have a measure for the area of the membrane that contacts the support, and thus of the size of the indented area of the eye.
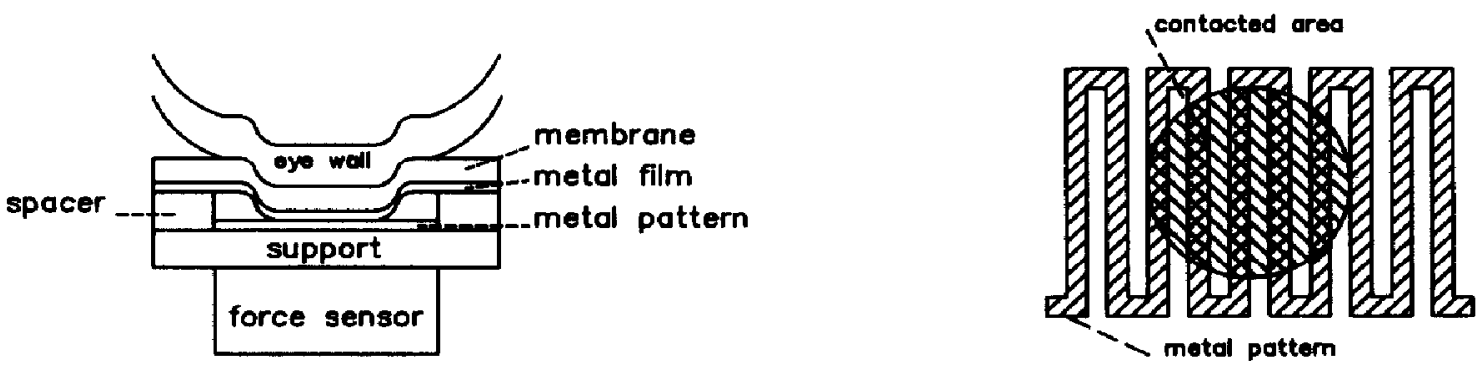

Fig. 2. Schematic drawing of the tonometric sensor (indentation of eye and membrane dimensions are not to scale).

Fig. 3. Top view of the sensor that measures the indented area.

\section{Experimental}

The sensors have been realized as follows. On a thermally-oxidized silicon wafer, a $150 \mathrm{~nm}$ layer of gold has been evaporated and photolithographically patterned. The length of the gold beams is $1 \mathrm{~cm}$ and the width $15 \mu \mathrm{m}$. The distance between two beams is also $15 \mu \mathrm{m}$, which means that the lowest detectable change in the radius of the indented area is equal to $30 \mu \mathrm{m}$. We have calculated that this is sufficient for an accuracy better than $96 \%$. As already mentioned, the gold beams are connected in such a way that a meander is realized, with outer dimensions of $1 \mathrm{~cm}^{2}$ and a total length of $3 \mathrm{~m}$. It will thus be clear that the photolithography has to be performed carefully. Around the edge of the meander a $5 \mu \mathrm{m}$ thick 
polyimide layer is deposited as a spacer between the membrane and silicon support. As a membrane on top of this spacer we have used a $12.5 \mu \mathrm{m}$ thick layer of Mylar, provided with a $150 \mathrm{~nm}$ thick gold layer on its inner side.

To test our devices we have realized an artificial eye by using a waterfilled balloon with negligible stiffness, placed in a rigid ball-shaped container with a $2.5 \mathrm{~cm}$ radius and a circular hole. The pressure in it is controlled by means of a water column.

Figure 4 presents the experimental results. We have drawn the measured force exerted on the eye as a function of the measured area, with the applied intraocular pressure as a parameter. In the ideal case the force will linearly increase for increasing values of the indented area. However, we observe considerable deviations from this linear behaviour. First of all we note that a threshold force of about $10 \mathrm{mN}$ is required before any contact between the membrane and support is observed. Secondly, we note that for an indented area of about $30 \mathrm{~mm}^{2}$, the applied force rapidly increases for a small increase in indented area.

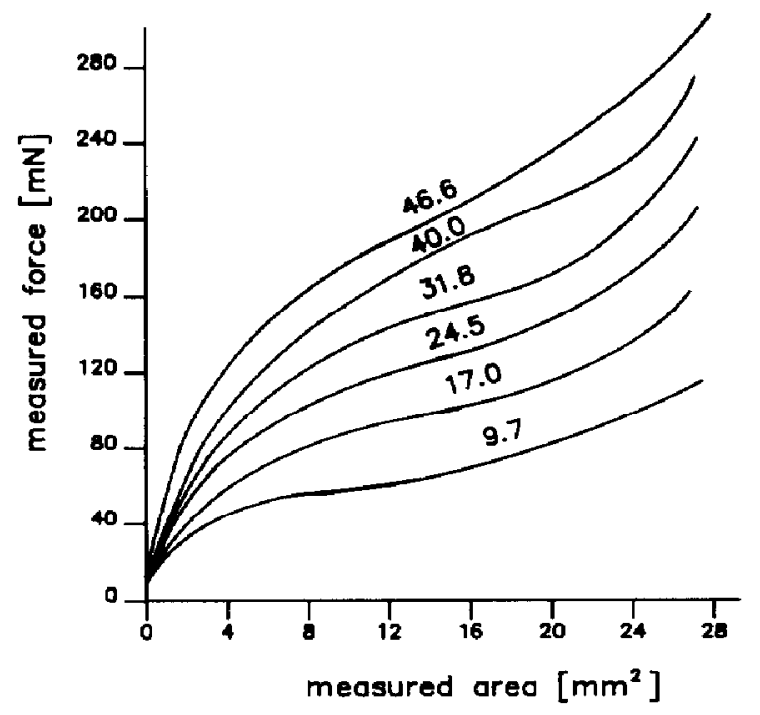

Fig. 4. Measured force as a function of the measured size of the contacted area, with the applied intraocular pressure as a parameter (in $\mathbf{m m ~} \mathbf{~ H g}$ ).

From the curves in Fig. 4 we have calculated the slope $d F / d A$, which is shown in Fig. 5 as a function of the measured area. Note that for a proper measurement, the slope of these curves has to be equal to the intraocular pressure $\left(P_{\text {iop }}=F / A\right)$. We observe, however, that the slopes are large for small as well as large values of the indented area, and that they are minimal for a measured area of about $15 \mathrm{~mm}^{2}$. We also see that for pressures larger than $25 \mathrm{~mm} \mathrm{Hg}$, the error of the measurements can be less than $10 \%$ if the minimum of the slope is taken as the intraocular pressure. 


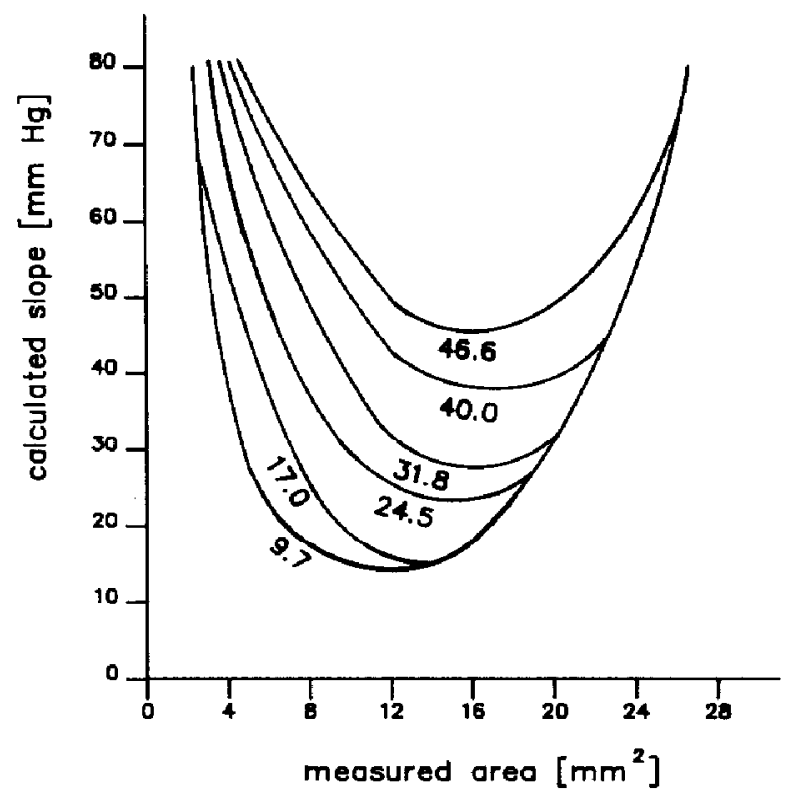

Fig. 5. Calculated slopes $d F / d A$ as a function of the contacted area, using the curves presented in Fig. 4, with the intraocular pressure as a parameter.

\section{Discussion and conclusions}

The observed threshold-force of about $10 \mathrm{mN}$ in Fig. 4 arises as follows. Before the membrane can contact the support, it has to deflect at least $5 \mu \mathrm{m}$ (the distance between the support and the unloaded membrane). This implies that a force has to be applied to the membrane, depending on its mechanical properties and dimensions. If the membrane and the support contact each other, the gold beams have to be short-circuited via the conductive gold film on the membrane. To surmount the contact resistance between this film and the gold meander, an additional force is required. Both these effects together mean that a small indentation of the eye (small force) will not result in a measurable contacted area via a change of resistance between the two ends of the meander.

The strong increase of the force as a function of measured area (Fig. 4) for values larger than $20 \mathrm{~mm}^{2}$ may be due to the stiffness of the membrane. The deflection of a membrane is strongly (fourth power) dependent on the radial distance from its centre. Therefore it is to be expected that for increasing values of the measured area, the required force to deflect that part of the membrane by at least $5 \mu \mathrm{m}$ strongly depends on the size of that part. This means that the difference between the indented area and the contacted area increases for increasing values of the contacted area. The measured area (via the resistance of the meander) is no longer equal to the indented area due to the mechanical properties of the membrane. 
Nevertheless, we observed that in taking the minimum value of the slope $\mathrm{d} F / \mathrm{d} A$ the error of the measurements is less than $10 \%$, especially for pressures larger than $25 \mathrm{~mm} \mathrm{Hg}$.

The average value of the intraocular pressure is about $20 \mathrm{~mm} \mathrm{Hg}$. Therefore it will be clear that the present sensor cannot be used and has to be improved. An important aspect will be the membrane behaviour. We have already found that a thinner membrane will result in a better sensor performance.

\section{References}

$1 \mathrm{H}$. Goldmann and Th. Schmidt, Über Applanationstonometrie, Ophthalmologica, 134 (1957) 221 - 242.

2 H. Goldmann and Th. Schmidt, Weitere Beitrag zur Applanationstonometrie, Ophthalmologica, 141 (1961) 441 - 456. 